\title{
License Plate Identification from Myanmar Vehicle Images under Different Environmental Conditions
}

\author{
Ohnmar Khin, ${ }^{1 *}$ Montri Phothisonothai, ${ }^{1}$ and Somsak Choomchuay ${ }^{2}$ \\ ${ }^{1}$ International College, King Mongkut's Institute of Technology Ladkrabang (KMITL), \\ Bangkok 10520, Thailand \\ ${ }^{2}$ Faculty of Engineering, King Mongkut's Institute of Technology Ladkrabang (KMITL), \\ Bangkok 10520, Thailand
}

(Received December 18, 2017; accepted August 28, 2018)

Keywords: car license plate recognition (CLPR), bounding box, horizontal and vertical dilation, skew angle detection, license plate detection, deep learning, neural networks

We have developed a license plate identification method for Myanmar vehicles that are captured under dissimilar conditions, e.g., angle of image capturing, different types of license plates, and real environmental conditions. In this study, car license plate recognition (CLPR), bounding box, horizontal and vertical dilations, skew angle detection, and plate detection were proposed to identify license numbers from different vehicle images. To recognize the characters, a new algorithm based on deep learning, a subset of artificial intelligence (AI), is proposed. The neural nets are progressing rapidly in many fields. The applied model of neural network is used for classification. The recognition part is a very challenging task. Compared with the traditional method, the neural network has obvious advantages. The benefit of this research is to eliminate the need of license plate recognition (LPR) under different conditions. In mobile phones, there are many sensors used to detect the presence of nearby objects. Accelerometers in mobile phones are used. Developed for the Samsung mobile phone, sensors can yield sensor readings but it not much else. Each car was viewed from four different angles under different conditions. In our experiment, the results showed an average accuracy of $97 \%$, which was substantially applied to license plate identification under different environmental conditions. To extend the experiment, the vehicle images were also collected under different conditions, such as dark and cloudy weather and various sizes and positions of plates.

\section{Introduction}

Vehicle license plate identification is essential in numerous situations in which it is important to distinguish vehicles for traffic control in unplaited areas, electronic payment of tolls on highways and bridges, and public security systems. License plate recognition (LPR) uses optical character recognition on vehicle images to read the license plates. LPR is being used in applications all over the world. The technology identifies and records the license plate numbers. Because there are various types of vehicles, the vertex of the plate from the ground varies depending on the car model; the plate may be located on the lower left or lower right part of the ${ }^{*}$ Corresponding author: e-mail: ohnmarkhin.cs@gmail.com https://doi.org/10.18494/SAM.2018.1852 
vehicle. Besides these variations in the placement of the plate on vehicles, the distance between the camera and the vehicles may also vary. This is important because the location of the plate inside the captured image plays a critical role. In this work, Myanmar-character and Englishcharacter license plates were studied. Nowadays, Myanmar-character plates are required to advance the related modern technologies.

The numbers 0-9 and 18 letters are used in Myanmar vehicle license plates. Myanmar letters and numbers include curves and straight lines, as shown in Fig. 1. Myanmar-character license plates are shown in Fig. 2. Images of dissimilar vehicles taken from four angles are shown in Fig. 3.

In Myanmar, according to its vehicle laws, either English or Myanmar characters can be used on license plates. Some examples are shown in Fig. 4. All license plates have the width of 14.1 inches $(35.8 \mathrm{~cm})$ and the height of 6.4 inches $(16.25 \mathrm{~cm})$. There is a white line boardering the plate. The difference between the license plates is the background color. The English characters are of the same size and they have the width of $1 \mathrm{~cm}$. All license plates include three stamps of the Ministry of Transportation.

There are seven types of English-character license plates in Myanmar. Both Myanmar and English-character license plates are shown in Fig. 4. Figure 4(a) shows a Myanmar-character taxi license plate with white characters on a red background. Figure 4(b) shows a United Nations vehicle plate with black characters on a white background. On this license plate, there are five characters: the first two characters are letters and others are numbers. Figures 4(c) and 4(d) show a religious vehicle plate with black characters on a yellow background and a hearse vehicle plate with white characters on a saddle brown background, respectively. These license plates have seven characters: the first three characters are letters and the others are numbers.

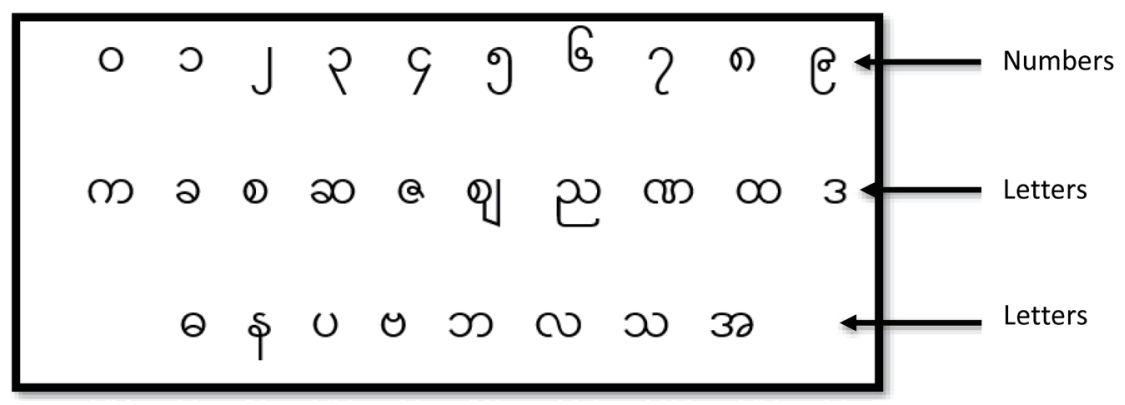

Fig. 1. Myanmar letters and numbers.

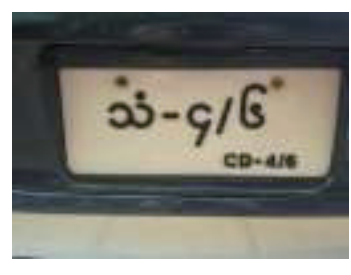

(a)

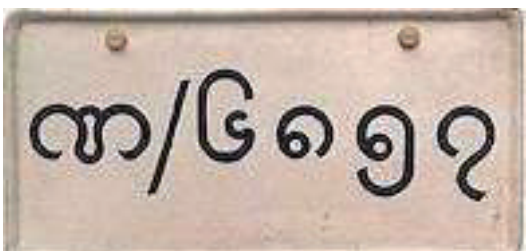

(b)

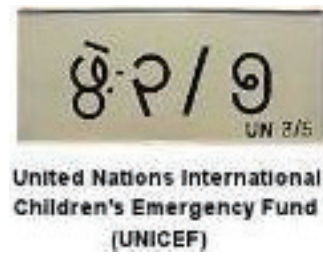

(c)

Fig. 2. (Color online) Myanmar-characters license plates. (a) Embassy vehicle, (b) private vehicle, and (c) United Nations vehicle. 

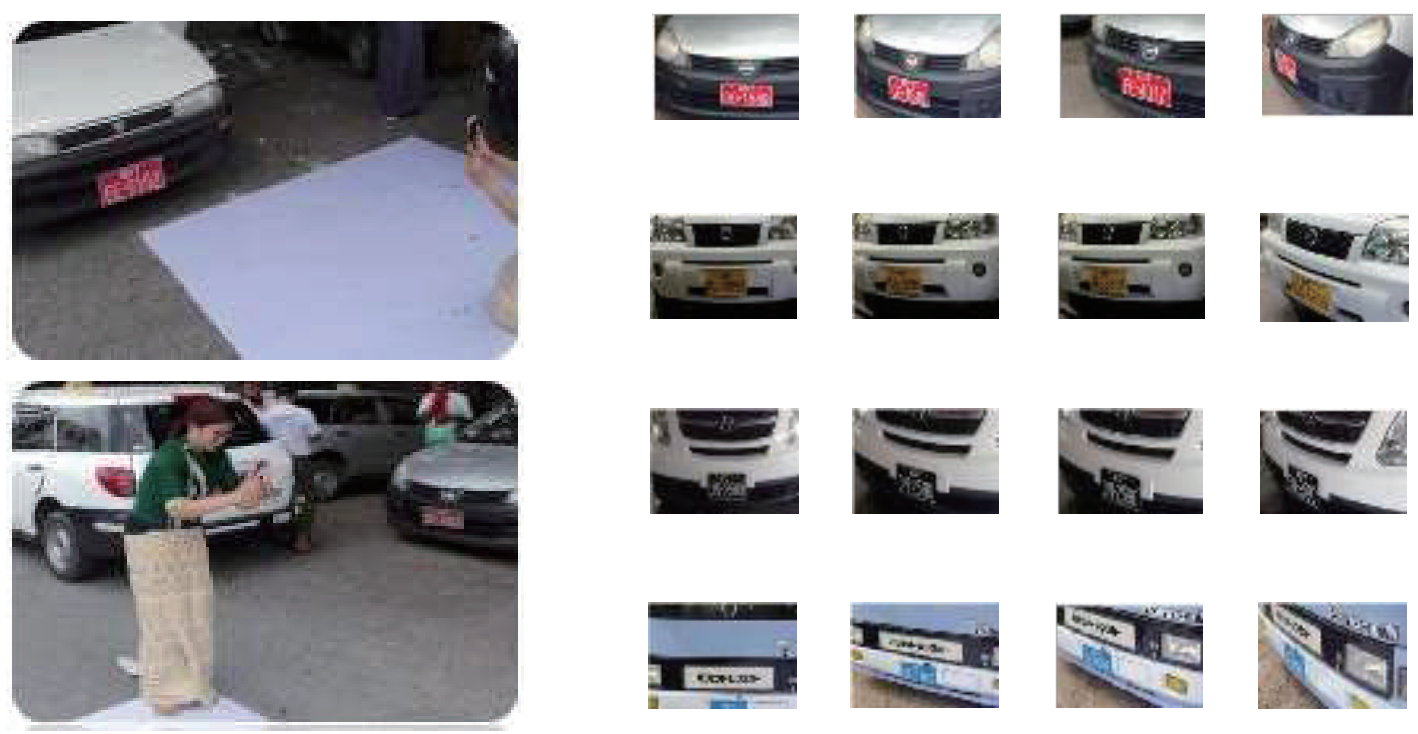

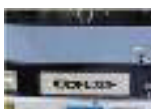

$90^{\circ}$

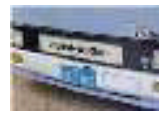

$60^{\circ}$

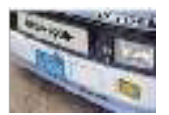

$45^{\circ}$

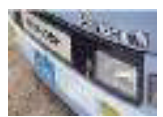

$30^{\circ}$

Fig. 3. (Color online) Images of dissimilar vehicles at four angles.

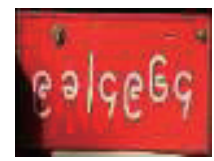

(a)

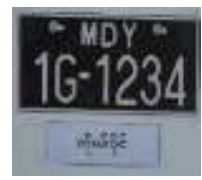

(e)

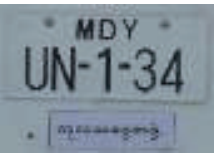

(b)

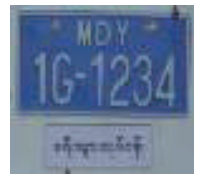

(f)

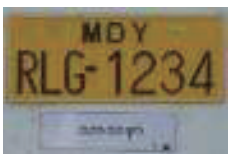

(c)

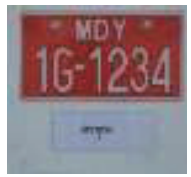

$(\mathrm{g})$

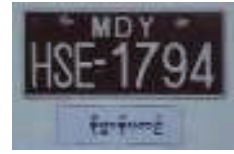

(d)

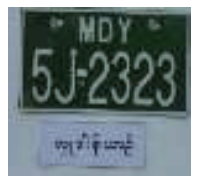

(h)

Fig. 4. (Color online) Types of license plate. (a) Myanmar-character license plate, (b) white plate for United Nations, (c) yellow plate for religious persons, (d) saddle brown plate for hearses, (e) black plate for private cars, (f) blue plate for foreigner tour buses, (g) red plate for taxies, and (h) green plate for donation. (http://myanmarrtad. com)

Figure 4(e)-4(h) show four plates: white characters on a black background for private cars, white characters on a blue background for tour buses for foreign tourists, white characters on a red background for taxies, and white characters on a green background for donation vehicles, respectively.

A great number of methods have been described in the literature. In the last year, localization and recognition of a Myanmar license plate on the basis of partially cut character structures were presented by Htay and Gopalakrishnan. ${ }^{(1)}$ The authors compared results in terms of advantages and disadvantages, processing speed, and accuracy. ${ }^{(1)}$ In Ref. 2, Kaur proposed a number plate extraction methodology. There are several traditional plate extraction techniques, such as Sobel edge detection, image subtraction, morphological operations, and 
thresholding. In Ref. 3, Shapiro et al. reported a license plate localization (LPL) component for a car license plate recognition (CLPR) system. They tested it with single-frame gray-level images that were captured at a specific time of day with weather conditions as input. They showed the plate localization method that provided the best results. In Ref. 4, base text-line construction and neural network were created by Shan for LPR. A new method of vehicle license plate recognition (VLPR) was shown in that paper. In Ref. 5, RamyaSri proposed the number plate localization system. In that system, edge detection and morphological operation were used. The algorithm was reliable and adjustable for different lighting conditions. Makaoui proposed LPL with morphological operations, wavelet transform, and projection operations. ${ }^{(6)}$

LPR: LPR acquires impressive performance with the fast development of deep learning. In Ref. 7, the LPL and recognition algorithms were proposed by Bulugu in a design for Tanzanian car plate numbers. Part of the image was extended to increase the readability of plate numbers, and a smoothing median filter and noise removal were used for easy development. In Ref. 8, Samra and Khalefah reported LPL with genetic algorithms and dynamic image processing techniques. The connected component analysis technique (CCAT) was aimed at detecting the target objects. The results confirmed the incorporation of memory and speed. It can be used in other problem domains related to the license plate problem. In Ref. 9, Gautam and Ahmed presented efficient fuzzy edge detection by the successive Otsu method. Some groups ${ }^{(10-15)}$ have given an issue on LPD. Generally, the proposed method is based on a support vector machine, adaptive morphological closing, a Riesz-fractional-based model, and a new zonebased model. The experiments require many attempts and long time. ${ }^{(16)}$ Otsu researched the thresholding method from gray-level histograms. The result was very effective for image processing. The LPD of an Indian vehicle based on the new zone-based algorithm was presented by Choudhury and Negi. The results showed effective performance for identified number recognition with an adaptive template matching system. ${ }^{(17)}$ LPD in video images was proposed by Elbamby et al. This novel algorithm can detect numerous lisence plates in an actual time for different sizes in an unfamiliar and complicated situation. ${ }^{(18)}$ Plate detection by a robust and efficient method for the motivation of accurately localizing vehicle license plates from a complex location in real time has been suggested. ${ }^{(19)}$ Miyata and Oka described a method for LPD in vehicle images. A support vector machine is utilized for the candidate area and the position of LPD. The average detection rate is about $90 \% .{ }^{(20)}$ The automatic vehicle LPD approach has been reported. This method has a 95\% classification accuracy rate. ${ }^{(21)}$ That research provided the LPD method, the critical process in LPR. The algorithm has been investigated and compared with each other regarding solidity and repeatability. ${ }^{(22)}$ The LPD with a sparse autoencoder was presented by Yang et al. They applied a novel method to vehicle LPD. The experimental results demonstrated the diverse types of LPD having great accuracy. ${ }^{(23)}$ The goal of the current work is to show the histogram-based edge processing algorithm. The system is investigated using MATLAB. The expressive performance has been investigated and the accuracy tested for dissimilar sets of input images. ${ }^{(24)}$ The automatic plate detection algorithm using corner features in video was reported by Patel et al. The Harris algorithm can identify license plates successfully from the input video. The advantages of the algorithm are less complex, high speed, and high quality. ${ }^{(25)}$ The experiments need many attempts and 
require much time. Moreover, the previous methods are not compatible with Myanmar license plates owing to the differences in license plate type and aspect ratio. License plates under different conditions have never been studied before.

\section{Materials and Methods}

In this study, the different license plates were examined from various acute angles between 0 and $90^{\circ}$. The geometry for obtaining $30,45,60$, and $90^{\circ}$ views was computed and is shown in Fig. 5. The system is intelligent enough to identify vehicle license plates viewed from those acute angles. Any camera can be used to take the images of license plates. There are many sensors in the mobile phones that are used to detect the presence of nearby plates.

Different environmental conditions make it difficult to identify a region of interest (ROI), i.e., a license plate in an original image by traditional methods. The general purpose of the recognition system is a very challenging task and errors in this step can make the algorithm inaccurate. This research focuses on Myanmar-character recognition. For the proposed algorithms, neural networks have obvious advantages. In this study, we proposed a method of dealing with different types of license plates. The experimental results are targeted for use in traffic-light development in Myanmar. This experimental paradigm has been designed to yield the top rates of convergence and properties of strength and good conditions of the solution.

In this investigation, license plate images were captured using the built-in camera of a smartphone as an alternative for LPR: Galaxy A7 Samsung smartphone with 5.5" full-highdefinition active-matrix organic light-emitting diode display, a full metal body, and six distinct colors. The camera has 13 megapixel resolution. Mobile phone sensors developed by Samsung can provide sensor readings.

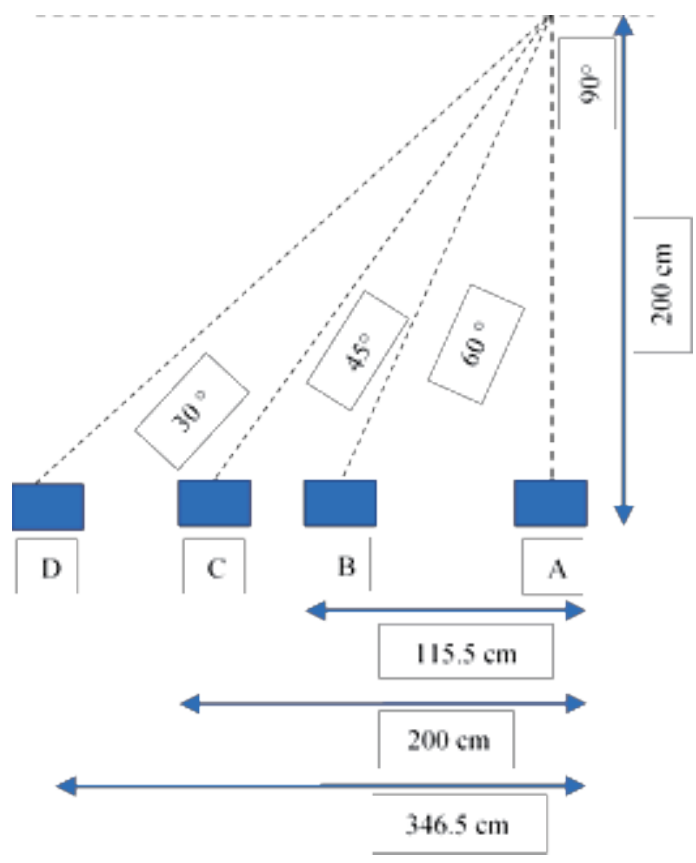

Fig. 5. (Color online) Experimental scheme of taking photographs from four different angles. 
VLPR involves the identification of vehicles from their license plates under various environmental conditions. The main steps of the process are image capture, license plate detection, character segmentation, and character recognition, as shown in Fig. 6. One of the most important tasks is license plate identification, which is defined as the finding of the exact location of the plate in the image. The flowchart of our proposed method is shown in Fig. 7. The captured images are converted into grayscale images by Otsu's method. The experimental identification results are shown in the following sections.

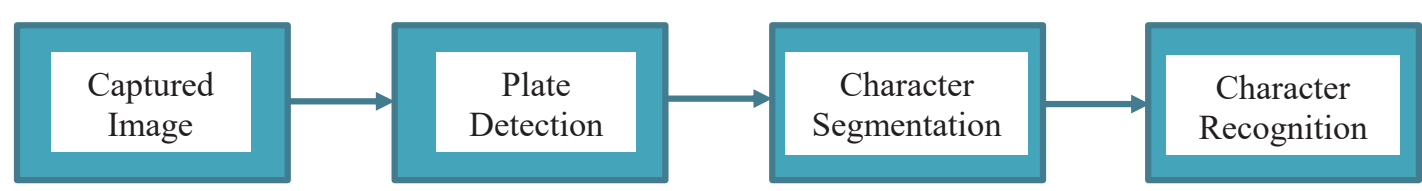

Fig. 6. (Color online) The main process.

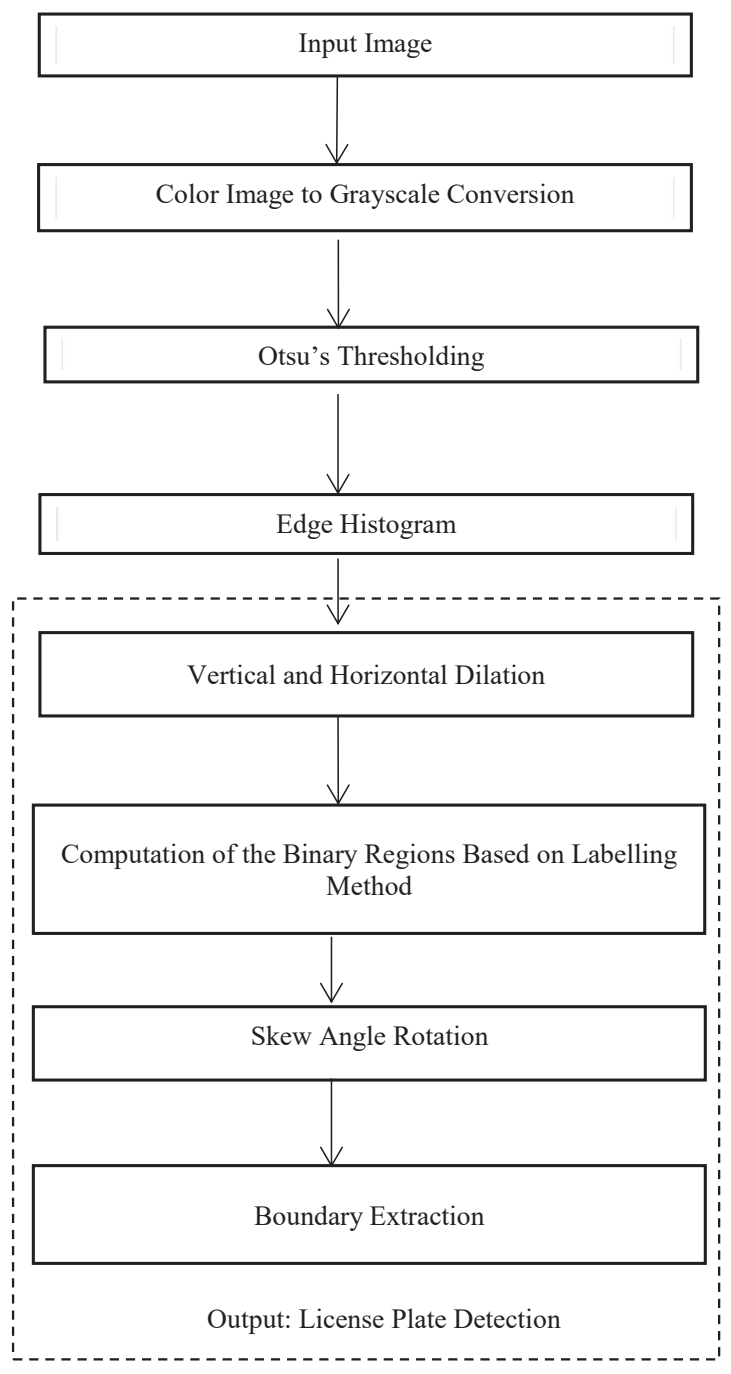

Fig. 7. Flowchart of main steps of proposed method. 


\section{Results and Discussion}

\subsection{Image acquisition}

Images of vehicles under various conditions were taken. The vehicles were photographed from a distance of $200 \mathrm{~cm}$. Plates having both Myanmar and English characters were photographed. Photographs were taken at angles of $90,60,45$, and $30^{\circ}$, as shown in Fig. 8. The Samsung's Galaxy A7 smartphones were used. The camera had 13 megapixel resolution. The photographs enabled the identification of the plates. The aim of this research is to detect the different angles of the license plate with the nonfixed LPR system.

\subsection{Conversion from RGB to grayscale}

Grayscale images were obtained from color images. The RGB image was composed of $30 \%$ red, $60 \%$ green, and $10 \%$ blue. In the process, the RGB image was converted to a grayscale image. The limits were between black and white values: 255 is white and 0 is black. The process alters the color to grayscale by eliminating hue and saturation information. It eliminates unnecessary information and noise. The resulting images are shown in Fig. 9.

\subsection{Thresholding (Otsu's method)}

There are two types of thresholding methods: global thresholding and local thresholding. Otsu's method is for global thresholding. It is widely used because it is simple and effective. We have classified Otsu's algorithm as a thresholding region-based segmentation algorithm. Also, the complexity of Otsu's algorithm is very high.

The threshold value requires evaluation. The best way to determine the best threshold for converting a color image to black and white is to look at the histogram of the image. The original image needs to be bright. The threshold must enable the separation of the license plate from background.

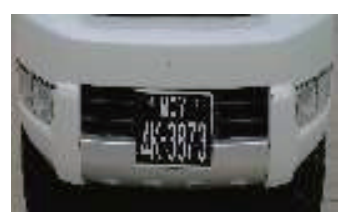

(a)

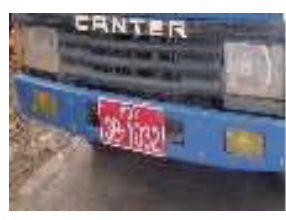

(b)

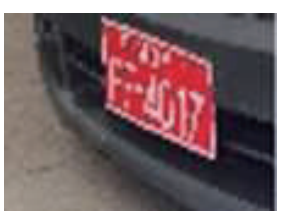

(c)

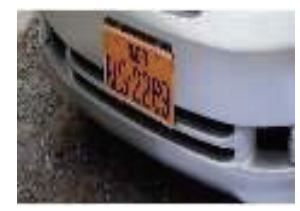

(d)

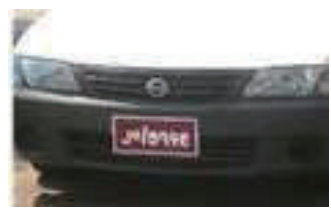

(e)

Fig. 8. (Color online) Vehicle license plate color images. (a) Private car plate at $90^{\circ}$, (b) truck plate at $60^{\circ}$, (c) taxi plate at $45^{\circ}$, (d) religious car plate at $30^{\circ}$, and (e) Myanmar-character car plate. 


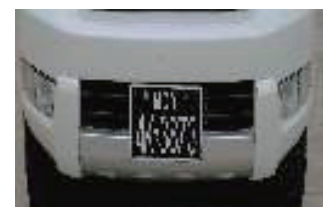

(a)

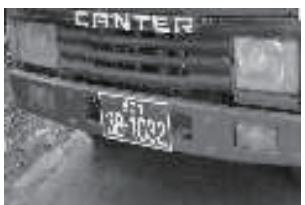

(b)

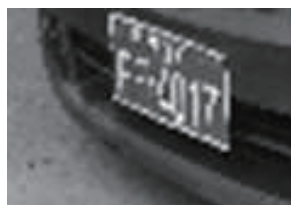

(c)

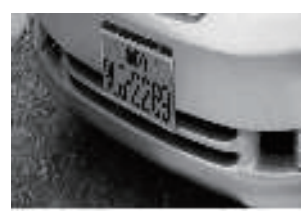

(d)

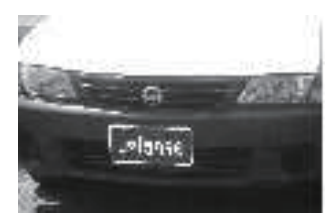

(e)

Fig. 9. Grayscale images. (a) Private car plate at $90^{\circ}$, (b) truck plate at $60^{\circ}$, (c) taxi plate at $45^{\circ}$, (d) religious car plate at $30^{\circ}$, and (e) Myanmar-character car plate.

Threshold algorithm: Otsu's method ${ }^{(16)}$ is described by

$$
\sigma_{w}^{2}(t)=w_{1}(t) \sigma_{1}^{2}(t)+w_{2}(t) \sigma_{2}^{2}(t)
$$

where the weights $w_{1}$ and $w_{2}$ are two categories, the threshold is $t$, and $\sigma_{1}{ }^{2}$ and $\sigma_{2}{ }^{2}$ are variations. The results of this step are shown in Fig. 10.

\subsection{Vertical and horizontal dilation}

The aim is to complete the dilation of the input image using the structuring element and then returning the dilated image. Traditional dilation uses fixed structuring elements. In other words, the resolution and model of a vehicle cannot be changed when the method is applied. Other uses of dilation are of practical interest because of its properties. ${ }^{(10)}$ This method specifies and determines the element of the district. The entire image is input, and then the dilation method identifies the plate area in a localized section. A considerable number of input images is applied in this process. If any of the pixels is set to 1 , the output pixels are also set to 1 in the binary image. From the exploratory results, the plate area are found to be accurately detected if the input image is of good quality. To identify the plate area, the dilation method is applied to the computation of the binary regions needed in the next step. The results of this step are shown in Fig. 11.

\subsection{Computing binary regions using labelling method}

The outcome of the dilation step is treated by the labelling method. This method is needed to enable the highly accurate location of the plate. 'Binarization' is the initial process and yields binarized images. The images include numerous things that are divided individually. We use this method to obtain the high region, which is an important feature for detecting license plates. The result of this method is shown in Fig. 12. 


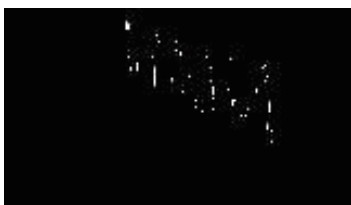

(a)

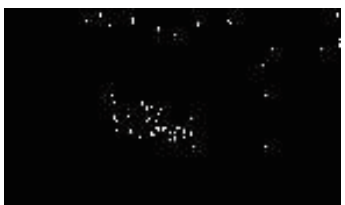

(b)

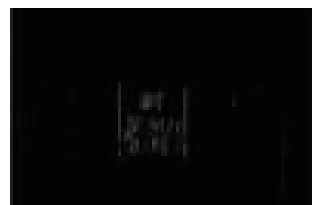

(c)

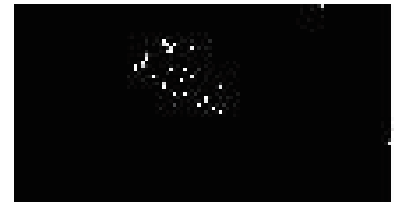

(d)

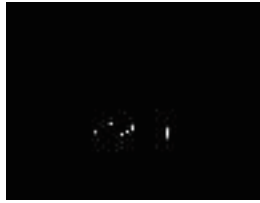

(e)

Fig. 10. Threshold images. (a) Private car plate at $90^{\circ}$, (b) truck plate at $60^{\circ}$, (c) taxi plate at $45^{\circ}$, (d) religious car plate at $30^{\circ}$, and (e) Myanmar-character car plate.

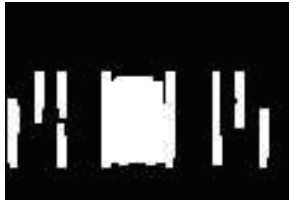

(a)

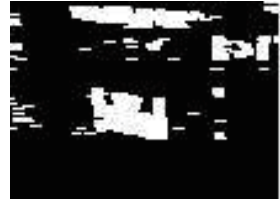

(b)

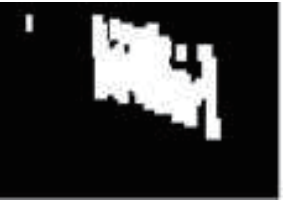

(c)

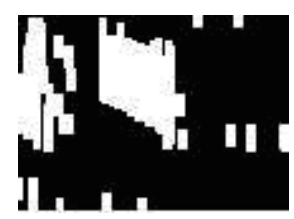

(d)

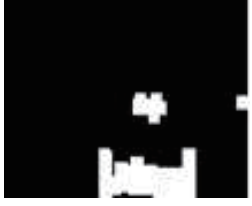

(e)

Fig. 11. Results of dilation of different images. (a) Private car plate at $90^{\circ}$, (b) truck plate at $60^{\circ}$, (c) taxi plate at $45^{\circ}$, (d) religious car plate at $30^{\circ}$, and (e) Myanmar-character car car plate.

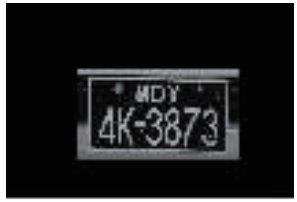

(a)

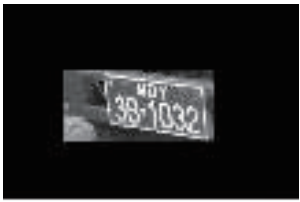

(b)

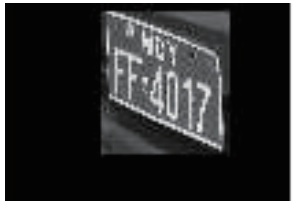

(c)

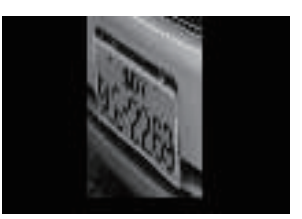

(d)

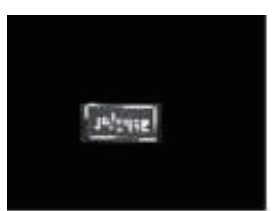

(e)

Fig. 12. Binary regions determined by labelling method for dissimilar images. (a) Private car plate at $90^{\circ}$, (b) truck plate at $60^{\circ}$, (c) taxi plate at $45^{\circ}$, (d) religious car plate at $30^{\circ}$, and (e) Myanmar-character car plate. 
The goal of the pseudocode is to find the clusters of similar pixels that are connected to each other. Labelling assigns a value to each pixel, defines similar values, and determines the pixels that are connected. Labelling also identifies the pixels of the plate area.

The pseudocode for integral images includes num, binary region BL, license plate L, column of input image $\mathrm{c}$, row of input image $\mathrm{r}$, index of input image $\mathrm{v}$, area $\mathrm{A}$, maximum $\mathrm{m}$, and largest binary region indexes $\mathrm{La}$ and $\mathrm{Lb}$.

The pseudocode for the labelling method is

1: procedure LM (c, r, L, A,)

2: $\mathrm{L}=\mathrm{BL}$

3: num $=\mathrm{BL}$

4: $\quad \mathrm{A}=0$

5: for $i=1$ to num

6: $\quad$ if $(\mathrm{L}==\mathrm{i})$ then

7: $\quad \mathrm{r}=\mathrm{L}$

8: $\mathrm{c}=\mathrm{L}$

9: $\quad \mathrm{v}=\mathrm{L}$

10: $A(i)=\operatorname{sum}(v)$

11: end if

12: if $($ Area $==\max ($ Area $)$ ) then

13: $\mathrm{La}=$ Area

14: $\mathrm{Lb}=$ Area

15: end if

16: end for

17: end procedure

\subsection{Skew angle detection}

The rotation function creates an output image that includes the entire input image. Similarly, this function uses nearest-neighbor interpolation to determine the value of pixels in the output image. We specify the image to be rotated and the rotation angle in degrees. The result of this step is shown in Fig. 13.

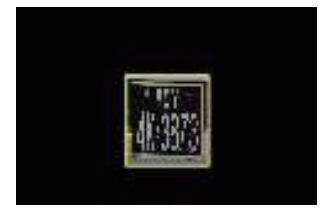

(a)

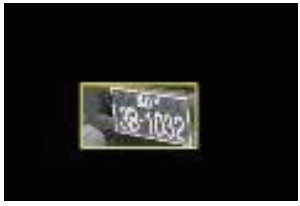

(b)

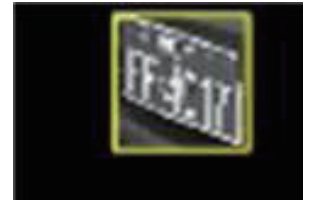

(c)

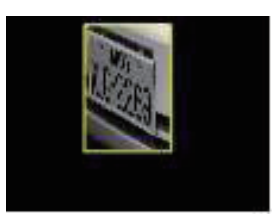

(d)

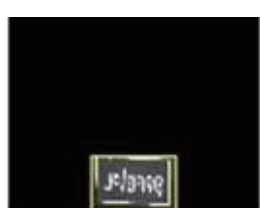

(e)

Fig. 13. Skew angle detection and correction. (a) Private plate at $90^{\circ}$, (b) truck plate at $60^{\circ}$, (c) taxi plate at $45^{\circ}$, (d) religious plate at $30^{\circ}$, and (e) Myanmar-character plate. 


\subsection{Actual number plate detection by boundary extraction}

The extraction method identifies the border of an object. To achieve more accurate results, the boundaries of the license plate area in an image are highlighted and the identification of the rectangles in the image is attempted.

Our proposed extraction method is based on the bounding box as follows. The function boundaries mark the objects in the vehicle image with a blue rectangle boundary. This proposed method is effective for different types of lisence plates.

Here, the symbols are extracted from the vehicle images, as shown in Fig. 14. The boundaries of objects in the images are analysed. To obtain more accurate results, the traced region is felled with pixels. The various positions of license plates are extracted by this process.

\subsection{Myanmar-character segmentation and extraction}

The input images are of Myanmar-character license plates. They contain characters of different sizes. As explained above, the input color images are converted into grayscale images. These images are binarized by a thresholding technique. Noise is removed from the images. All the objects containing less than 30 pixels are removed. Then, further processing is executed on these images.

To segment and extract the characters of the license plate, the image processing toolbox function is used. There are many steps in this process. In object extraction, the foreground objects are extracted from the image.

The features are extracted using bounding boxes in the area. Bounding boxes in an image are used to represent a possible license plate ROI. The algorithm returns the ROI in the form of pixel coordinates and the width and height. The pixels inside the image are connected. If the area of this connected component is greater than the threshold area, then, its bounding box is stored and is displayed near the text region. The text is detected but includes some incorrect

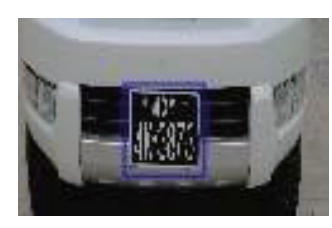

(a)

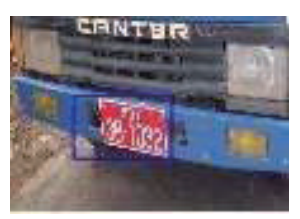

(b)

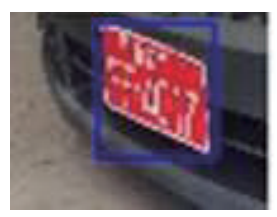

(c)

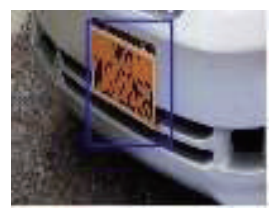

(d)

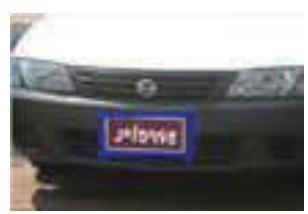

(e)

Fig. 14. (Color online) Actual number plate detection by boundary extraction. (a) Private car plate at $90^{\circ}$, (b) truck plate at $60^{\circ}$, (c) taxi plate at $45^{\circ}$, (d) religious car plate at $30^{\circ}$, and (e) Myanmar-character car plate. 
extraction. From the segmented image, the characters and numbers are extracted one by one. The text is detected but there is some incorrect extraction. The extraction results of letters and numbers are shown in Fig. 15.

\subsection{Myanmar-character recognition}

For plate recognition, Myanmar characters are focused on because the recognition of English characters has already been successfully completed in other previous work. Mostly, character recognition is based on the template matching method, which requires much effort and time. In addition, the algorithms are not compatible with Myanmar license plates owing to differences in color, plate aspect ratio, and characters. Therefore, a deep learning, a powerful set of techniques for a neural network, was designed. This is obviously advantageous to detection accuracy.

As explained above, Myanmar license plates have characters and numbers. Myanmar characters consist of many curves. In addition, most characters and numbers are similar in shape to each other. Eighteen letters and 10 numbers are used for recognition. We choose data clash to make the recognition network powerful. In the preprocessing, each character image is normalized into $32 \times 32$ and the grayscale image is transformed into a binary image to reduce the complexity. From the binary image, the feature values of 18 letters and 10 numbers $(28$ classes) are extracted for the neural network. The extracted features are shown in Table 1. The neural network adopted in this study is composed of input layers, hidden layers, and output layers, as shown in Fig. 16. Most neural approaches are based on combinations of elementary processors, each of which takes several inputs and generates a single output. Each input is associated with a weight, and the output is then a function of the weighted sum of inputs; this output function may be discrete or continuous, depending on the type of network in use. A simple neuron is shown in Fig. 17. The simple neuron is described as

$$
x=\sum_{i}^{n} v_{i} w_{i}
$$

The inputs denoted as $v_{1}, v_{2}, v_{3}, \ldots$, and the weights as $w_{1}, w_{2}, w_{3}, \ldots$, are the total input to the neuron.

For character recognition, the number $n$ of input units $X$, the number $m$ of output units $Y$, and the number $l$ of hidden units $Z$ are used. The number of input units ( $n$ ) is 144 (neurons), the number of output units $(m)$ is 28 (neurons), and the number of hidden units $(1)$ is 144 (neurons).
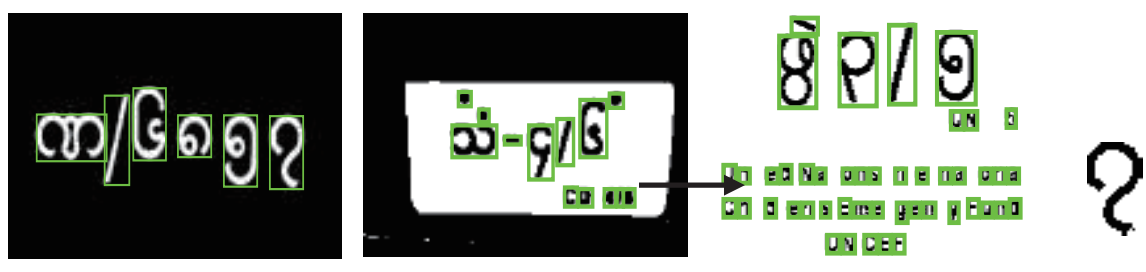

Fig. 15. (Color online) Letters and numbers extraction. 
Table 1

Feature extraction.

\begin{tabular}{|c|c|c|c|c|c|c|c|c|c|c|}
\hline \multirow{2}{*}{$\begin{array}{l}\text { Class (18 letters } \\
\text { and } 10 \text { numbers) }\end{array}$} & \multicolumn{10}{|c|}{ Extracted features } \\
\hline & Area & Diameter & Eccentricity & Histogram & Contrast & Correlation & Homogeneity & Texture & Skeleton & Regions \\
\hline $\mathrm{O}$ & 17 & 4.652 & 0.871 & 0.433 & 0.496 & 69.121 & -0.013 & 0.006 & 0.284 & 11 \\
\hline o & 19 & 4.918 & 0.858 & 0.511 & 0.500 & 202.376 & 0.155 & 0.003 & 0.180 & 12 \\
\hline J & 12 & 3.909 & 0.888 & 0.419 & 0.494 & 56.974 & 0.133 & 0.007 & 0.237 & 8 \\
\hline p & 17 & 4.652 & 0.871 & 0.5 & 0.501 & 71.7 & 0.045 & 0.005 & 0.233 & 11 \\
\hline q & 17 & 4.652 & 0.871 & 0.338 & 0.473 & 85.807 & -0.012 & 0.007 & 0.252 & 11 \\
\hline Э & 87 & 10.525 & 0.835 & 0.435 & 0.496 & 75.586 & 0.029 & 0.006 & 0.241 & 32 \\
\hline G & 481 & 24.747 & 0.890 & 0.699 & 0.459 & 148.927 & 0.087 & 0.002 & 0.186 & 53 \\
\hline 2 & 97 & 11.113 & 0.797 & 0.583 & 0.493 & 215.181 & 0.003 & 0.002 & 0.159 & 34 \\
\hline a & 17 & 4.652 & 0.871 & 0.343 & 0.475 & 84.818 & -0.016 & 0.007 & 0.249 & 11 \\
\hline e & 7 & 2.985 & 0.853 & 0.501 & 0.501 & 80.405 & -0.247 & 0.005 & 0.198 & 5 \\
\hline$m$ & 17 & 4.652 & 0.871 & 0.613 & 0.487 & 261.773 & -0.008 & 0.002 & 0.152 & 11 \\
\hline ว & 86 & 10.464 & 0.836 & 0.431 & 0.495 & 199.177 & 0.133 & 0.003 & 0.148 & 33 \\
\hline (1) & 13 & 4.068 & 0.884 & 0.426 & 0.495 & 84.804 & -0.079 & 0.006 & 0.231 & 8 \\
\hline$\infty$ & 19 & 4.918 & 0.858 & 0.598 & 0.491 & 317.007 & -0.053 & 0.002 & 0.152 & 12 \\
\hline a & 18 & 4.787 & 0.858 & 0.429 & 0.495 & 98.333 & -0.092 & 0.006 & 0.205 & 12 \\
\hline D & 17 & 4.652 & 0.871 & 0.493 & 0.500 & 194.965 & 0.071 & 0.003 & 0.164 & 11 \\
\hline حు & 17 & 4.652 & 0.871 & 0.508 & 0.500 & 178.481 & 0.006 & 0.002 & 0.163 & 11 \\
\hline ๘) & 17 & 4.652 & 0.871 & 0.531 & 0.499 & 206.174 & -0.048 & 0.002 & 0.157 & 11 \\
\hline$\infty$ & 17 & 4.652 & 0.871 & 0.489 & 0.500 & 198.188 & 0.148 & 0.003 & 0.176 & 11 \\
\hline 3 & 16 & 4.514 & 0.837 & 0.547 & 0.498 & 215.877 & 0.024 & 0.001 & 0.156 & 10 \\
\hline$\theta$ & 23 & 5.412 & 0.858 & 0.471 & 0.499 & 285.425 & -0.050 & 0.003 & 0.139 & 13 \\
\hline$\$$ & 17 & 4.652 & 0.871 & 0.491 & 0.500 & 195.081 & 0.058 & 0.003 & 0.156 & 11 \\
\hline 0 & 17 & 4.652 & 0.871 & 0.633 & 0.482 & 253.707 & -0.023 & 0.002 & 0.152 & 11 \\
\hline y & 19 & 4.918 & 0.858 & 0.419 & 0.494 & 339.623 & -0.049 & 0.003 & 0.138 & 12 \\
\hline$\infty$ & 13 & 4.068 & 0.862 & 0.406 & 0.491 & 190.812 & 0.042 & 0.004 & 0.154 & 9 \\
\hline o & 19 & 4.918 & 0.858 & 0.429 & 0.495 & 87.939 & -0.116 & 0.006 & 0.214 & 12 \\
\hline యి & 18 & 4.787 & 0.858 & 0.433 & 0.496 & 186.404 & 0.157 & 0.003 & 0.153 & 12 \\
\hline 32 & 22 & 5.293 & 0.861 & 0.469 & 0.499 & 289.875 & -0.028 & 0.003 & 0.137 & 14 \\
\hline
\end{tabular}

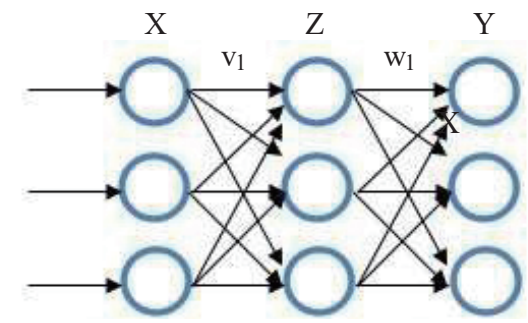

Fig. 16. (Color online) Neural network.

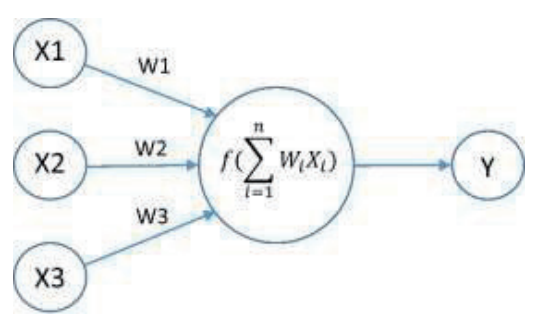

Fig. 17. (Color online) Simple neuron.

Each neuron is a transfer function $f(x)$, as described in Eqs. (3)-(5).

$$
\begin{aligned}
& f(x)=0, \text { if } x \leq 0 \\
& f(x)=1, \text { if } x>0 \\
& f(x)=\frac{1}{1+e^{-x}}
\end{aligned}
$$


A threshold is associated with the neuron with transfer function $f(x)$, which provides the output.

To improve the identification accuracy, we use data argument. The training dataset includes 28 classes. The verification dataset includes over 50 vehicle images. The training is iterated 315 times. The width of the image is $36.2 \mathrm{~cm}$, while the height is $16.5 \mathrm{~cm}$. The recognition result after training is shown in Table 2. The average recognition success rate of each character is $97.74 \%$. Table 2 also shows the numbers of characters recognized. Characters not detected are shown in Fig. 18.

\section{Discussion}

Many techniques for LPD have been developed. The previous methods involved vehicle LPL and comparison of results. The current system can detect license plates viewed from an angle smaller than a right angle. In reality, LPR must be done for plates viewed from various angles. Therefore, an effective method must accomodate different angle conditions.

The experiments were performed on the images taken as part of traffic-light development work in Myanmar. Over 100 vehicle images were recorded under different angle conditions. To obtain images with the four different angles of $90,60,45$, and $30^{\circ}$, we use the configuration described in Fig. 4. Point $\mathrm{A}$ is at a position perpendicular to the license plate at a distance of $200 \mathrm{~cm}$. Point $B$ is on the same plane as point $A$ at a distance of $115.5 \mathrm{~cm}$ from point A. Point $\mathrm{C}$ is $200 \mathrm{~cm}$ from point $\mathrm{A}$ and point $\mathrm{D}$ is $346.5 \mathrm{~cm}$ from point $\mathrm{A}$.

To extend the experiment, vehicle images are also acquired under different conditions of darkness, cloudiness, size, position, resolution, color of plates, and distance. The images are shown in Table 3.

We found that the image size is a significant feature. We experimented with image sizes of $1904 \times 916$ and $2322 \times 4128$. The image sizes are shown in Table 4 . In the experiment, the image size of $1904 \times 916$ is used. Using the small size of $151 \times 120$ gave an incorrect result. It was also found that the detection rate was affected by the brightness and clarity of the vehicle and the license plate. The parameter values used in the proposed method are shown in Table 5. The variables in this experiment are displayed in Table 6 and the results of performance evaluation are shown in Tables 7-10.

Table 2

Number of characters recognized and success rate.

\begin{tabular}{lcccc}
\hline & Class & Total number & Success numbers & Success rate (\%) \\
\hline Numbers & 10 & 25 & 23 & 92.00 \\
Letters & 18 & 108 & 107 & 99.07 \\
Total & 28 & 133 & 130 & 97.74 (average) \\
\hline
\end{tabular}

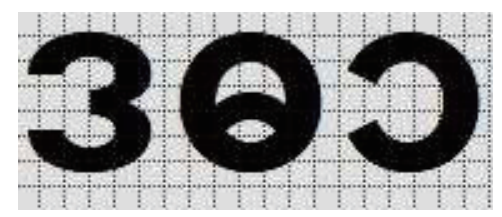

Fig. 18. Characters not detected. 
Table 3

Results of proposed technique.

Results for different conditions

Different sizes and positions

Different types of license plates
Cloudiness and darkness
Various angles

Table 4

Experimental image size.

\begin{tabular}{lll}
\hline Methods & Conditions & Image size \\
\hline & Angle of $90^{\circ}$ & $2322 \times 864$ pixels \\
Horizontal and vertical dilation, & Angle of $60^{\circ}$ & $2322 \times 1263$ pixels \\
Labelling method, & Angle of $45^{\circ}$ & $1946 \times 1095$ pixels \\
Skew angle detection, & Angle of $30^{\circ}$ & $2180 \times 974$ pixels \\
Bounding box & Cloudy and dark & $1024 \times 518$ pixels \\
& Various types of license plates & $2322 \times 974$ pixels \\
& Various sizes and positions & $2048 \times 1536$ pixels \\
\hline
\end{tabular}

Table 5

Parameter values.

\begin{tabular}{lc}
\hline Parameter name & Parameter value/type \\
\hline Threshold on edge histogram & $T=0.1$ \\
Vertical dilation & $70 \times 70$ pixels \\
Horizontal dilation & $8 \times 95$ pixels \\
Plate region by T pixel & $T=10$ \\
\hline
\end{tabular}

Table 6

Variables in the experiment.

\begin{tabular}{lc}
\hline Angles & $90,60,45,30^{\circ}$ \\
\hline Colors of plates & Black, yellow, blue, red \\
Car colors & Black, white, blue, red, yellow \\
Light condition & Normal, cloudy, darker \\
\hline
\end{tabular}

Table 7

Results of performance evaluation in the case of $90^{\circ}$.

\begin{tabular}{lccccc}
\hline Conditions & \multicolumn{5}{c}{ Experimental results } \\
\hline Light condition & Normal 97\% & Cloudy 90\% & Darker 87\% & & \\
Different colors of plates & B-W 97\% & Y-B 97\% & B-W 97\% & R-W 97\% & \\
Different car colors & Black 97\% & White 97\% & Blue 97\% & Red 97\% & Yellow 97\% \\
\hline
\end{tabular}

Table 8

Results of performance evaluation in the case of $60^{\circ}$.

\begin{tabular}{lccccc}
\hline Conditions & \multicolumn{5}{c}{ Experimental results } \\
\hline Light condition & Normal 91\% & Cloudy 87\% & Darker 87\% & \\
Different colors of plates & B-W 90\% & Y-B 90\% & B-W 90\% & R-W 90\% & \\
Different car colors & Black 90\% & White 90\% & Blue 90\% & Red 90\% & Yellow 90\% \\
\hline
\end{tabular}


Table 9

Results of performance evaluation in the case of $45^{\circ}$.

\begin{tabular}{lccccc}
\hline Conditions & \multicolumn{5}{c}{ Experimental results } \\
\hline Light condition & Normal 97\% & Cloudy 90\% & Darker 87\% & \\
Different colors of plates & B-W 97\% & Y-B 97\% & B-W 97\% & R-W 97\% & \\
Different car colors & Black 97\% & White 97\% & Blue 97\% & Red 97\% & Yellow 97\% \\
\hline
\end{tabular}

Table 10

Results of performance evaluation in the case of $30^{\circ}$.

\begin{tabular}{lccccc}
\hline Conditions & \multicolumn{5}{c}{ Experimental results } \\
\hline Light condition & Normal 91\% & Cloudy 87\% & Darker 87\% & \\
Different colors of plates & B-W 90\% & Y-B 90\% & B-W 90\% & R-W 90\% & \\
Different car colors & Black 90\% & White 90\% & Blue 90\% & Red 90\% & Yellow 90\% \\
\hline
\end{tabular}

Table 7 shows the experimental results of vehicle detection in the case of $90^{\circ}$. The results in Table 9 show no difference compared with those in Table 7. These results indicate satisfactory accuracy of vehicle detection. The reason is that Table 9 has only one identification and the system can detect it.

In contrast, Tables 8 and 10 show the results for vehicle identification with viewing angles of 30 and $60^{\circ}$. The detection accuracy decreases in these cases. However, our system can detect vehicles in various input images with satisfactory results. For the recognition accuracy, refer back to Table 2.

For plate detection, the dataset consists of 100 real images photographed at a traffic light. The performance of our proposed approach was examined regarding detection rate, character extraction, and character recognition, which are defined below.

- Detection rate: The number of complete plates extracted. All characters of the extracted license plate should be visible and there should be significant number regions.

- Character extraction: The letters and numbers are extracted one by one from different plates. The calculated results for the extracted characters are shown.

- Character recognition: For high accuracy, the neural network for character recognition is adopted. The proposed method is evaluated and found to yield satisfactory outcomes.

Furthermore, to validate the proposed identification method, we conduct experiments with view angles of $90,60,45$, and $30^{\circ}$ by the bounding boxes method. The proposed method gives good results.

In the cases of $90,60,45$, and $30^{\circ}$, the methods, including the proposed method, yield low results for light contribution compared with normal data because the algorithm affects the clearance of the vehicle. Cloudy weather is the worst optimal for sunlight. For plate identification, a fairly clear day is preferred.

Default parameter values are evaluated when the program is compiled. It is an important distinction. Parameters are variables. The threshold value, dilation value, and plate region by pixel value affect the results.

When the threshold value is large, the algorithm cannot extract the area of the plate from the vehicle image. It is important to identify the plate area. According to the results, the plate areas 
Table 11

Results of license plate detection.

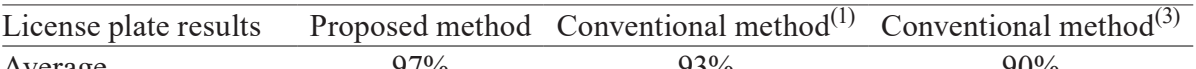

\begin{tabular}{llll}
\hline Average & $97 \%$ & $93 \%$ & $90 \%$
\end{tabular}

are successfully detected if the input image is clear. The dilation method requires labelling to compute the binary regions in the next step. In this process, labelling indicates the high region as the important feature in detecting the plates. The horizontal and vertical default parameter values are $70 \times 70$ and $8 \times 95$ pixels, the amounts of change of the dilation effect are $30^{\circ}$ and less than $30^{\circ}$, and the extracted areas of the plates are large and small, respectively. The skew angle detection rotates the angle in degrees. The method for extraction is the bounding box method. This method specifies the objects within the plate region, which is a rectangle with its boundary marked in blue, by their $\mathrm{T}$ pixel value on the vehicle image. The smaller value more strongly affects the algorithm. We cannot achieve extraction using the smaller value. The larger value has little effect on the change.

The numerous methods are indicative of the extensive research on character segmentation and recognition. The images of vehicles such as cars and motorcycles are segmented and reconditioned in this research. The current extraction methods focus on both uppercase numbers and letters and degrade in the case of lowercase characters. However, our proposed method is consistent for all datasets with few differences. Recognition failed for some characters because the experimental sample was poor. In our next research, we will examine an efficient character recognition method.

The advantage of our proposed method is that verification is possible through comparison with previous experimental results. We tested the vehicle images obtained by conventional methods with the same parameters and by our system.

The proposed method is compared with the conventional method, ${ }^{(1)}$ in which Myanmar LPL and recognition are implemented using the partially cut character structure. The results for three methods are given in Table 11. In the conventional method, $92.7 \%$ of license plate characters are recognized. Results obtained in previous work ${ }^{(3)}$ involving filtering, edge detection, and slope evaluation are compared with the results of another conventional method for license plate extraction. Despite the daylight conditions, there are portions of the plate that are shadowed, leading to failure of detection. The accuracy of the method is $90 \%$.

\section{Conclusions}

The purpose of this study was to develop and evaluate an acceptable technique for the identification of Myanmar vehicle license plates under different conditions. The experimental results showed an excellent ability of our method to detect license plates under different conditions. To verify our new method, we tested it using vehicle images. Detection may be impossible if the color of the car body is identical to that of the license plate or if the size of the license plate is very small compared with the whole vehicle. We observed that preprocessing can solve this problem. In preprocessing, each character image is normalized, and a grayscale 
image is transformed into a binary image to lower the complexity. The results obtained using our new method indicated better performance than the conventional method.

Our system was evaluated using captured vehicle images by offline analysis. The time taken to detect the license plate in vehicle images using MATLAB 2016b software was $19 \mathrm{~s}$. The overall character recognition rate with our method was over $90 \%$, showing a very good performance. However, the number of experimental samples was very small. Therefore, we used general block letters instead of letters on the number plate image. The neural network enabled the recognition of most of the characters and numbers, even those very similar to each other. Recognition of some characters failed. In the next research, we will pursue a more efficient recognition technique to improve the overall performance and reliability.

\section{Acknowledgments}

This investigation was supported by King Mongkut's Institute of Technology Ladkrabang, Thailand. I extend heartfelt thanks to my advisors for their support, supervision, and advices. I deeply thank my advisor, Associate Professor Dr. Somsak Choomchuay, for teaching, helping, and encouraging me to finish this paper. He thoroughly oversaw this research until the last date. This paper is dedicated to him.

\section{References}

1 M. M. Htay and A .K. Gopalakrishnan: 14th Int. Conf. ICT and Knowledge Engineering (ICT\&KE) (2016) 38.

2 S. Kaur: Int. J. Comput. Sci. Inf. Technol. 5 (2014) 2954.

3 V. Shapiro, D. Dimov, S. Bonchev, V. Velichkov, and G. Gluhchev: Int. Conf. Comput. Syst. Technol. (CompSysTech') (2003) IIIA.2-7.

4 B. Shan: J. Comput. 6 (2011) 246.

5 A. P. RamyaSri : Int. J. Innovations Eng. Technol. 2 (2013) 200.

6 K. Makaoui, Z. Guennoun, and M. Ghogho: 2nd Int. Conf. Electr. Inf. Technol. (ICEIT) (2016). https://doi. org/10.1109/EITech.2016.7519628

7 I. Bulugu: Int. J. Sci. Res. 2 (2013) 12.

8 A. Samra and F. Khalefah: IEEE Trans. Evol. Comput. 18 (2014) 244.

9 D. Gautam and M. Ahmed: Int. Conf. Convergence in Technology (2014) 1-5.

10 B. A. Fomani and A. Shahbahrami: 3rd Int. Conf. Pattern Recognition and Image Analysis (IPRIA) (2017) 146.

11 A. C. Roy, M. K. Hossen, and D. Nag: Int. Conf. Electrical Engineering and Information \& Communication Technology (iCEEiCT) (IEEE, 2016). https://doi.org/10.1109/CEEICT.2016.7873098

12 K. S. Raghunandan, P. Shivakumara, H. A. Jalab, R. W. Ibrahim, G. H. Kumar, U. Pal, and T. Lu: IEEE Trans. Circuits Syst. Video Technol. 28 (2018) 1.

13 S. Miyata and K. Oka: 14th Int. Conf. Control, Autom. Rob. \& Vision, Phuket, Thailand (ICARCV) (2016).

14 G. L. Corneto, F. A. Silva, D. R. Pereira, L. L. Almeida, A. O. Artero, J. P. Papa, V. H. C. de Albuquerque, and H. M. Sapia : IEEE Lat. Am. Trans. 15 (2017) 75.

15 A. Choudhury and A. Negi: Fourth Int. Conf. Parallel, Distributed and Grid Computing (PDGC) (2016) 370374.

16 N. Otsu: IEEE Trans. Syst. Man Cybern. 9 (1979) 62.

17 A. Choudhury and A. Negi: 4th Int. Conf. Parallel, Distributed and Grid Computing (PDGC) (2016) 370.

18 A. Elbamby, E. E. Hemayed, D. Helal, and M. Rehan: 12th Int. IEEE Computer Engineering Conf. (IEEE, ICENCO) (2016) 148-153.

19 Y. Yuan, W. Zou, Y. Zhao, X. Wang, X. Hu, and N. Komodakis: IEEE Trans. Image Process. 26 (2017) 1102.

20 S. Miyata and K. Oka: 14th Int. Conf. Control, Autom. Rob. Vision, Phuket, Thailand (2016) 1-5.

21 G. L. Corneto, F. A. Silva, D. R. Pereira, L. L. Almeida, A. O. Artero, J. P. Papa, V. H. C. de Albuquerque, and H. M. Sapia: IEEE Lat. Am. Trans. 15 (2017) 75. 
22 K. Horak, J. Klecka, and P. Novacek: 39th Int. Conf. Telecommunications and Signal Processing (TSP) (IEEE, 2016) 484-488.

23 R. Yang, H. Yin, and X. Chen: 8th Int. Symp. Computational Intelligence and Design (2015) 465.

24 S. Chhabra, H. Jain, and S. Saini: Int. Conf. Advances in Computing, Communications and Informatics (ICACCI) (2016) 1181-1187.

25 H. N. Patel, K. Desai, and T. Panchal: Int. Conf. Inf. Process. (ICIP) (2015) 301.

\section{About the Authors}

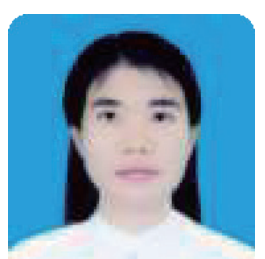

Ohnmar Khin received her B.S. degree from Yatanabon University, Myanmar, in 2008 and her M.Sc. degree from Yangon University, Myanmar, in 2011. Since 2016, she has been studying for her master's degree in computing in engineering systems at King Mongkut's Institute of Technology Ladkrabang, Bangkok, Thailand, under the AUN/SEED-Net scholarship. Her research interests are in computer vision and image processing.

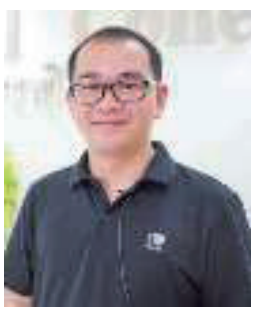

Montri Phothisonothai received his B.S. and M.Eng. from King Mongkut's University of Technology Thonburi (KMUTT), Thailand, in 2002 and 2004, respectively. In 2008, he received his Ph.D. in Information Science and Control Engineering, Nagaoka University of Technology, Japan, through the 21st Center of Excellence (COE), MEXT Scholarship. From 2013-2015, he also served as an associate editor of the Journal of Integrative Neuroscience, Imperial College Press. He held a Japan Society for the Promotion of Science (JSPS) Fellowship at the University of Tokyo during 2011-13. Currently, he is a lecturer at International College, King Mongkut's Institute of Technology Ladkrabang (KMITL), Thailand. His research interests are information processing, biomedical signal processing, brain-machine interaction, and cognitive science. He is a member of ECTI, Thailand.

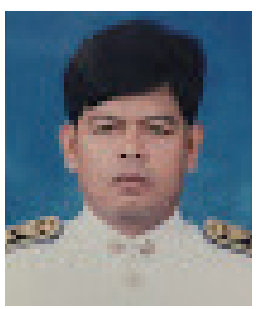

Somsak Choomchuay received his M.Phil. and Ph.D. from Imperial College, University of London, United Kingdom in 1994, after which he joined King Mongkut's Institute of Technology Ladkrabang, Bangkok, Thailand. He was promoted to associate professor of electronic engineering in 2000. His current research interests are data security, error control codes, and applications of signal processing in biomedical engineering. Dr. Somsak is a member of ECTI (Thailand) and IEEE (USA). 